\title{
Proof the Skewes' number is not an integer using lattice points and tangent line
}

\author{
V. ĎURIŠ, T. ŠUMNÝ AND T. LENGYELFALUSY
}

\begin{abstract}
Skewes' number was discovered in 1933 by South African mathematician Stanley Skewes as upper bound for the first sign change of the difference $\pi(x)-\operatorname{li}(x)$. Whether a Skewes' number is an integer is an open problem of Number Theory. Assuming Schanuel's conjecture, it can be shown that Skewes' number is transcendental. In our paper we have chosen a different approach to prove Skewes' number is an integer, using lattice points and tangent line. In the paper we acquaint the reader also with prime numbers and their use in RSA coding, we present the primary algorithms Lehmann test and Rabin-Miller test for determining the prime numbers, we introduce the Prime Number Theorem and define the prime-counting function and logarithmic integral function and show their relation.
\end{abstract}

Mathematics Subject Classification 2000: 11P21, 11G99

Keywords: Skewes' number, proof, Prime Number Theorem, prime-counting function, logarithmic integral function, lattice points, tangent line

\section{INTRODUCTION}

The prime numbers play an essential role in mathematics, e.g. they are the cornerstone of modern cryptographic algorithms and protocols, such as digital signatures, public key encryption, etc. The basic attribute of prime numbers is that they are no longer decomposable into the product of other numbers, and this very attribute is important for modern cryptography. In number theory we know several algorithms, by means of which we can decompose composite numbers into the product of prime numbers while we will use the same algorithm to decompose multidigit numbers as for example to decompose small numbers. The results of the mathematical research to date show that there is no general rule according to which it is possible to quickly decompose large numbers into prime numbers universally and that the process of decomposition of large numbers takes a long time even for modern computer technology.

Thus, we can easily algorithmically decompose only numbers of a certain "small" size into the product of prime numbers and this fact is the basis of virtual security. If we take two very large prime numbers, where both the first and the second 
represent some information, it is easy to multiply them with each other, giving a very large composite number practically decomposable (unless we know the so-called private key, i.e. some necessary information how the large number came up). Algorithms that would look for the factors of a product by trying all possibilities, would have an NP complete time complexity [1]

This security, using prime number attributes, allows for public key encryption [2]. It is an encryption in which each of its principles is known and is public, but no one decrypts the encrypted information, because the public key only (i.e. knowledge of the encryption principle) is not enough. To decipher the cipher and read the message, it is necessary to obtain prime number elements of prime factorization that can only be obtained by the recipient of the message who also knows the private key (that is, some necessary information on how the product originated).

There are currently several encryption algorithms that use prime factorization, but historically the most important is RSA [3]. One of the first standards was RSA768 [4], which represents a 232-digit number. The standard has been broken by scientists by bringing together hundreds of computers that have been working for a period representing 2000 years of one computer work. Later, the RSA-1024, RSA2048 or RSA-4096 standards have been developed which are used today.

All virtual security builds on exceptional properties of prime numbers, and that is the reason we are still studying prime numbers with great importance. Prime numbers are the basic building blocks of all numbers, and much of the modern knowledge comes only from discovery of other prime number properties.

\section{GENERATING PRIME NUMBERS}

Many modern cryptographic algorithms and protocols require prime numbers, so it is important to be able to properly generate them and be able to effectively decide whether a given number $n$ is a prime. Probability of randomly selected number near the number $n$ being the prime number is approximately $\frac{1}{\ln n}$ [5]. Then the total number of primes less than $n$ is approximately $\frac{n}{\ln n}$ (more in Section 3 ).

When generating prime numbers, a number $n$ is randomly selected and one of the known prime number tests is used to determine if it is prime. If not, another 
random number $n$ can be selected and repeat the test. Number $n$ must be selected appropriately (e.g. in the form of $2^{p}-1$, where $p$ is a prime number [6]) to increase the probability of choosing a prime number.

One of the known tests, e.g. Lehmann test [7] or Rabin-Miller test [8] can be used for prime number testing. Lehmann's prime number test of $n$ is an algorithm consisting of the following steps:

1. we choose a random number $m$ less than $n$

2. we calculate $m^{\frac{n-1}{2}} \bmod n$

3. if $m^{\frac{n-1}{2}} \not \equiv 1(\bmod n)$ or $m^{\frac{n-1}{2}} \not \equiv 1(\bmod n)$, the number $n$ is not a prime number

4. if $m^{\frac{n-1}{2}} \equiv 1(\bmod n)$ or $m^{\frac{n-1}{2}} \equiv-1(\bmod n)$, the probability of $n$ not being a prime is maximum $50 \%$

The algorithm is repeated $k$-times with another randomly selected number $m$, and if the test succeeds $k$-times, the probability of $n$ not being prime, will be $m \frac{1}{2^{k}}$.

Rabin-Miller's prime number test is a very fast probability algorithm which, if it finds that a given number is composite, then it is really composite. If the algorithm in response returns that number $n$ is a prime number, it's only true with a certain probability. Rabin-Miller's prime number test of $n$ is an algorithm consisting of the following steps:

1. first for a random number $n$ we find such a number $u$, so that $2^{u} \mid n-1$, thus $n-1=2^{u} \cdot l$, where $l$ is an odd number

2. then we select a random number $m$, so that $1<m<n$ applies

3. for each $i=0, \cdots, u-1$ we count $v_{i}=m^{2^{i} \cdot l} \bmod n$, and if $i$ exists for which does not apply that $\left(v_{i}=-1 \vee m^{l} \bmod n=1\right)$, then $n$ is composite

4. if $n$ is not composite, we repeat steps $2-3 k$ times

5. if after repeating the test $k$ times $n$ is not composite, it can be a prime number

The probability of passing the test by a composite number as a prime decreases faster in this test and is equal to $\frac{1}{4^{k}}$. Thus, by repeating the test multiple times, we can 
reduce the probability of an error to arbitrarily small one, though never zero, and we cannot say with certainty that the algorithm has returned a prime number.

Sometimes, e.g. in RSA cryptographic algorithms using the product of two prime numbers $p, q$, prime numbers are required to be strong prime numbers [9]. These prime numbers have certain properties that make it difficult to decompose a number into prime factors using standard procedures. Recommended properties include:

1. the largest common divisor of numbers $(p-1)$ and $(q-1)$ should be small

2. both numbers $(p-1)$ and $(q-1)$ should have great prime factors $r, s$

3. both numbers $(r-1)$ and $(s-1)$ should have great prime factors

4. both numbers $(r+1)$ and $(s+1)$ should have great prime factors

5. both numbers $\frac{(p-1)}{2}$ and $\frac{(q-1)}{2}$ should be prime numbers

\section{PRIME-COUNTING FUNCTION AND THE PRIME NUMBER THEOREM}

The prime-counting function, denoted as $\pi(x)$, is the function that counts the number of prime numbers less than or equal to a given real number $x$ [10]:

$$
\pi(x)=\sum_{p \leq x} 1
$$

In the history of mathematics, there have been many attempts to find the exact formula for prime-counting function [11-12]. In the end of the $18^{\text {th }}$ century French mathematician Adrien-Marie Legendre conjectured that

$$
\pi(x) \sim \frac{x}{A \cdot \log (x)+B}
$$

for constants $A$ and $B(\log (x)$ denotes the natural $\operatorname{logarithm})$ in his work [13] which was published in 1798 and summarized the number theory results of the $18^{\text {th }}$ century. Legendre improved his conjecture in 1808 in his work [14] to

$$
\pi(x)=\frac{x}{\log (x)-A(x)}
$$


where $\lim _{x \rightarrow \infty} A(x)=1.08366$ is Legendre's constant [15]. Carl Friedrich Gauss followed up on the research of Legendre from the $18^{\text {th }}$ century and in 1849 he brought a better estimate for $\pi(x)$ based on empirical proofs obtained from the tables of prime numbers as:

$$
\lim _{x \rightarrow \infty} \frac{\pi(x)}{\frac{x}{\log (x)}}=1, \operatorname{resp} . \pi(x) \sim \frac{x}{\log (x)}
$$

In 1854, a professor at St. Petersburg University, Pafnuty Lvovich Chebyshev, found approximation $0.92129<\frac{\pi(x)}{\frac{x}{\log (x)}}<1.10555$ for function $\pi(x)$. When studying function $\pi(x)$, Chebyshev used a real function which in the complex domain in its form $\zeta(z)=\sum_{n=1}^{\infty} \frac{1}{n^{z}}$ is known under the name Riemann function (zeta function) defined in the whole complex plane except from point 1 . This function had already been known to Euler in the $18^{\text {th }}$ century but it was only Bernhard Riemann who fully discover its potential. Riemann tried to find nontrivial zero values of function $\zeta(z)$ during his attempt to use function $\zeta$ to prove formula $\lim _{x \rightarrow \infty} \frac{\pi(x)}{\frac{x}{\log (x)}}=1$. In 1859 he formulated a conjecture, the Riemann Hypothesis, [16] that in a plane of complex numbers $z=x+\mathrm{i} y$ in a planar strip defined by inequality $0 \leq x \leq 1$, all these zero points lie on line $x=\frac{1}{2}$. A decision on the validity of Riemann hypothesis would solve a large number of problems from various areas of mathematics, especially from the number theory domain, such as the question of prime number distribution. Riemann zeta function contains an infinite number of zeros and, by using available numerical methods, by 1986 it was proven that 1,500,000,000 zeros of Riemann zeta function lies on line $x=\frac{1}{2}$. Based on current calculations, this holds for up to 10 quintillions of zeros.

The theorem $\lim _{x \rightarrow \infty} \frac{\pi(x)}{x / \ln x}=1$ in known as the Prime Number Theorem and it is one of the most remarkable results of modern mathematics. The theorem was proved simultaneously in 1896 by Hadamard [17] and de la Vallée-Poussin [18]. Hence the Prime Number Theorem shows that around any number $x$ the density of prime 
numbers is about $\frac{1}{\log (x)}$. Several values for $\pi(x)$ are shown in the Table 1 together with the actual number of prime numbers up to $x$ [19].

$\begin{array}{ccc}x & \begin{array}{c}\text { number of primes less } \\ \text { than or equal to } x\end{array} & \pi(x) \\ 1000 & 168 & 178 \\ 10000 & 1229 & 1246 \\ 100000 & 9592 & 9630 \\ 1000000 & 78498 & 78628 \\ 10000000 & 664579 & 664918 \\ 100000000 & 5761455 & 5762209 \\ 1000000000 & 50847534 & 50849235 \\ 10000000000 & 455052511 & 455055614\end{array}$

Table 1. Several values for $\pi(x)$

The Prime Number Theorem is equivalent to statement:

$$
\pi(x) \sim \operatorname{li}(x)
$$

where $\operatorname{li}(x)$ li is the logarithmic integral function [20] defined for all positive real numbers $x>1$ as:

$$
\operatorname{li}(x)=\int_{0}^{x} \frac{d t}{\ln t}
$$

The function $\frac{1}{\ln t}$ has a singularity at $t=1$, and the function $\operatorname{li}(x)$ is interpreted as a Cauchy principal value [21]:

$$
\operatorname{li}(x)=\lim _{\varepsilon \rightarrow 0^{+}}\left(\int_{0}^{1-\varepsilon} \frac{d t}{\ln t}+\int_{1+\varepsilon}^{x} \frac{d t}{\ln t}\right) .
$$

It was thought for a long time that $\pi(x)$ is always an overestimate for all $x$ :

$$
\pi(x)<\operatorname{li}(x)
$$

until John Edensor Littlewood, a British mathematician, showed in 1914 [22] that the logarithmic integral function underestimates the prime counting function for some large $x(\pi(x)>\operatorname{li}(x))$. Moreover that the difference $\pi(x)-\operatorname{li}(x)$ changes sign 
infinitely often. Littlewood's proof did not state a concrete such number $x$, it was just a proof of existence.

The first crossover point (as upper bound for the first sign change) was discovered by South African mathematician Stanley Skewes (Skewes was supervised for his PhD. under the Littlewood) and this number was named after him. In 1933 [23] Skewes showed there exists a number $x$ below:

$$
e^{e^{e^{79}}}<10^{10^{10^{34}}}
$$

This bound assumed that the Riemann Hypothesis is true. In 1955 [24] Skewes showed a bound

$$
e^{e^{e^{e^{7.705}}}}<10^{10^{10^{964}}}
$$

without assuming the Riemann hypothesis known as Skewes Second Number.

\section{MAIN RESULTS}

Consider a space with a rectangular coordinate system $[O, x, y]$. Coordinate axes $x, y$ as numeric axes have integer values highlighted. If by these highlighted points we draw lines perpendicular to the considered axis we get a grid in which the node point is defined as $A\left[x_{0}, y_{0}\right], x_{0}, y_{0} \in \mathbb{Z}$. In the work [25] we have shown a method to find solutions for a certain type of Diophantine equations using a tangent line (plane). We use the slope of a tangent line and its shift on the relevant axis to determine the properties of the tangent point and whether it is a grid point or not.

We now apply this procedure to determine whether the considered number $b$ belongs to the set of integers or not. Consider a function $y=f(x)$ under the condition $y=f\left(x_{0}\right)=b$ and $x_{0} \in \mathbb{Z}$. Then if the tangent line at the point $E\left[x_{0}, b\right]$ has a rational slope and at the same time a shift on the relevant axis $s_{x}$ at the point $B\left[s_{x}, 0\right]$ is rational, number $b \in \mathbb{Z}$. In the case of a more complex number where we cannot provide the condition $x_{0} \in \mathbb{Z}$, we consider $x_{0}=\alpha \cdot \delta$ where $\alpha \in \mathbb{R}$ and $\delta \in \mathbb{Z}$. Then the slope will be in the form $k=\frac{1}{\alpha} \cdot q$ and $q \in \mathbb{Q}$. By such compensation of the grid, we can abolish the irrationality of the slope, which arose on the basis of the number 
we have chosen. Hence we get to the previous condition. In more computationally difficult tasks we also use the intersection with the $y$-axis. Then we use a line on which we have three points $A\left[0, s_{y}\right], B\left[s_{x}, 0\right]$ and $E\left[x_{0}, b\right]$.

We also use the gradient of field in our approach. The derivative of a scalar field $U=U(\vec{r})$ according to the vector $\vec{c}$ at a given point $\vec{r}$ is called the limit

$$
\frac{\partial U}{\partial \vec{c}}=\lim _{\varepsilon \rightarrow 0} \frac{U(\vec{r}+\varepsilon \vec{c})-U(\vec{r})}{\varepsilon} .
$$

If we consider the unit vector $\vec{c}_{0}$, equality holds

$$
\frac{\partial U}{\partial \vec{c}}=|\vec{c}| \cdot \frac{\partial U}{\partial \overrightarrow{c_{0}}}
$$

where $\frac{\partial U}{\partial \overrightarrow{c_{0}}}$ means the rate at which the function $U$ increases in the direction of the vector $\overrightarrow{c_{0}}$ at each point. The gradient of the field $\operatorname{grad}(U)$ is the vector defined at each point of the field and that has the direction of the normal line to the equiscular plane. In a right-angled coordinate system

$$
\operatorname{grad}(U)=\frac{\partial U}{\partial x} \vec{\imath}+\frac{\partial U}{\partial y} j+\frac{\partial U}{\partial \vec{z}} \vec{k}
$$

From the above properties we can determine based on the $\operatorname{grad}(U)$ equations of the tangent plane to the area determined by the equation of the scalar field $U$, or in the case of a situation in space $E_{2}$ we have a tangent line to the curve. More information on the theory can be found in [26-27].

Now, we'll show through lattice points and tangent line that Skewes' number is not an integer.

\section{PROBLEM. $e^{e^{e^{79}}} \in \mathbb{N}$}

PROOF. To prove that the Skewes' number is an integer, we use the property of a tangent line, which we place in the grid system. In this case, the slope of the tangent line depends on the properties of the function, with which we can create a mapping of our considered number to the number $e^{e^{e^{79}}}$. Consider a coordinate system $x y$ where we have defined the grid by $y=k_{1}, x=k_{2} \cdot e ; k_{1}, k_{2} \in \mathbb{Z}$. It is important for us that 
the grid is integer graduated on the $y$-axis, and due to the chosen function, we consider multiples of the number $e$ on the $x$-axis. We choose this form of the line for the calculation:

$$
(O-X) \cdot \operatorname{grad}(F[A])=D
$$

where $O$ is the origin of the coordinate system, $X$ is any point of the line, $E$ is the tangent point with the coordinates $E\left[x_{0}, y_{0}\right]$ and $D$ is the result of an inner product which depends on the distance of the line from the origin of the coordinate system.

For a line that does not pass through the origin of the coordinate system, we choose points that lie on the coordinate axes. Then the point of the line which lies on the coordinate axis $y$ has the coordinates $A\left[0, s_{y}\right]$ and the point which lies on the coordinate axis $x$ has the coordinates $B\left[s_{x}, 0\right]$. Now we solve the vector equation

$$
\begin{gathered}
(O-A) \cdot \operatorname{grad}(F[A])=D \\
\left(0-0,0-s_{y}\right) \cdot \operatorname{grad}(F[A])=D
\end{gathered}
$$

Next we multiply the equation by the vector $\operatorname{grad}(F[A])^{-1}$

$$
\begin{gathered}
\left(0,-S_{y}\right)=D \\
(O-B) \cdot \operatorname{grad}(E)=D \cdot \operatorname{grad}(F[A])^{-1}
\end{gathered}
$$

We need to determine the intersection with the $y$-axis by multiplying the equation by the unit vector $1_{y}=(0,1)$ and we get

$$
-S_{y}=D \cdot 1_{y} \cdot \operatorname{grad}(F[A])^{-1} .
$$

Next consider the curve given by the equation

$$
y=x^{x^{x^{79}}}
$$

We simplify the equation to the form $F(x, y)=0$. With a suitably chosen number for $x$, we are able to get to the required number. 
Now consider a tangent line at a point $A\left[e, e^{e^{e^{79}}}\right]$, which leads us to the number $e^{e^{e^{79}}}$, whose properties we are determining. Hence $\frac{y-y_{0}}{x-x_{0}}=\frac{e^{e^{e^{79}}}}{D \cdot 1_{x} \cdot \operatorname{grad}(F[A])^{-1}+e}$ and

$$
\begin{gathered}
\frac{y-y_{0}}{x-x_{0}}=\frac{D \cdot 1_{y} \cdot \operatorname{grad}(F[A])^{-1}+e^{e^{e^{79}}}}{e} \\
\frac{y-y_{0}}{x-x_{0}}=e^{e^{e^{79}}}\left(e^{-1+e^{79}}+e^{e^{79}}\left(e^{78}+79 e^{78}\right)\right) \\
D=e^{e^{e^{79}}}\left(e^{-1+e^{79}}-1+e^{e^{79}}\left(e^{78}+79 e^{78}\right)\right)
\end{gathered}
$$

Let's determine the tangent line based on the intersections with the coordinate system and the tangent point

$$
\begin{gathered}
\frac{s_{y}-y_{0}}{0-x_{0}}=\frac{0-y_{0}}{s_{x}-x_{0}} \\
\operatorname{grad}(F[A])^{-1}=\frac{\operatorname{grad}(F[A])}{\sqrt{\operatorname{grad}(F[A]) \cdot \operatorname{grad}(F[A])}} \\
\operatorname{grad}(F[A])=\left(e^{e^{e^{79}}}\left(e^{-1+e^{79}}+e^{e^{79}}\left(e^{78}+79 e^{78}\right)\right), 1\right)
\end{gathered}
$$

From that

$$
\begin{gathered}
\operatorname{grad}(F[A])^{-1}=\frac{\left(e^{e^{e^{79}}}\left(e^{-1+e^{79}}+e^{e^{79}}\left(e^{78}+79 e^{78}\right)\right), 1\right)}{\sqrt{\left(\left(e^{e^{79}}\right)^{2}\left(e^{-1+e^{79}}+e^{e^{79}}\left(e^{78}+79 e^{78}\right)\right)^{2}+1\right)}} \\
\frac{e^{e^{e^{79}}}}{D \cdot 1_{x} \cdot \operatorname{grad}(F[A])^{-1}+e}=\frac{D \cdot 1_{y} \cdot \operatorname{grad}(F[A])^{-1}+e^{e^{e^{79}}}}{e}
\end{gathered}
$$

After simplification it holds

$$
\begin{gathered}
e^{e^{e^{79}}+1}=\left(D \cdot 1_{x} \cdot \operatorname{grad}(F[A])^{-1}+e\right) \cdot\left(D \cdot 1_{y} \cdot \operatorname{grad}(F[A])^{-1}+e^{e^{e^{79}}}\right) \\
e^{e^{e^{79}}}=\frac{\left(D \cdot 1_{x} \cdot \operatorname{grad}(F[A])^{-1}+e\right) \cdot\left(D \cdot 1_{y} \cdot \operatorname{grad}(F[A])^{-1}+e^{e^{e^{79}}}\right)}{e}
\end{gathered}
$$


If $e^{e^{e^{79}}} \in \mathbb{N}$ then suppose that $e^{e^{e^{79}}}=k_{3} \in \mathbb{N}$. Then

$$
\left(D \cdot 1_{x} \cdot \operatorname{grad}(F[A])^{-1}+e\right) \cdot\left(D \cdot 1_{y} \cdot \operatorname{grad}(F[A])^{-1}+k_{3}\right)=k_{3} \cdot e .
$$

Hence

$$
\begin{gathered}
k_{3} \cdot e=D \cdot 1_{x} \cdot \operatorname{grad}(F[A])^{-1} \cdot D \cdot 1_{y} \cdot \operatorname{grad}(F[A])^{-1}+D \cdot 1_{x} \cdot \operatorname{grad}(F[A])^{-1} k_{3}+ \\
+e \cdot D \cdot 1_{y} \cdot \operatorname{grad}(F[A])^{-1}+e \cdot k_{3}
\end{gathered}
$$

From that

$$
\begin{gathered}
D \cdot 1_{x} \cdot \operatorname{grad}(F[A])^{-1} \cdot D \cdot 1_{y} \cdot \operatorname{grad}(F[A])^{-1}+D \cdot 1_{x} \cdot \operatorname{grad}(F[A])^{-1} k_{3}+ \\
+e \cdot D \cdot 1_{y} \cdot \operatorname{grad}(F[A])^{-1}=0
\end{gathered}
$$

After substitution we get

$$
\begin{gathered}
\frac{\left(\mathrm{k}_{3}\left(e^{-1+e^{79}}-1+\mathrm{k}_{3}\left(e^{78}+79 e^{78}\right)\right)\right)^{3}}{\left(\left(\mathrm{k}_{3}\right)^{2}\left(e^{-1+e^{79}}+e^{e^{79}}\left(e^{78}+79 e^{78}\right)\right)^{2}+1\right)}+ \\
+\frac{\left(\mathrm{k}_{3}\left(e^{-1+e^{79}}-1+\mathrm{k}_{3}\left(e^{78}+79 e^{78}\right)\right)\right)^{2} k_{3}+e\left(\mathrm{k}_{3}\left(e^{-1+e^{79}}-1+\mathrm{k}_{3}\left(e^{78}+79 e^{78}\right)\right)\right)}{\sqrt{\left(\left(\mathrm{k}_{3}\right)^{2}\left(e^{-1+e^{79}}+e^{e^{79}}\left(e^{78}+79 e^{78}\right)\right)^{2}+1\right)}} \\
=0
\end{gathered}
$$

And finally we have a form

$$
\begin{aligned}
& \left(\mathrm{k}_{3}\left(e^{-1+e^{79}}-1+\mathrm{k}_{3}\left(e^{78}+79 e^{78}\right)\right)\right) \\
& \cdot\left(\frac{\left(\mathrm{k}_{3}\left(e^{-1+e^{79}}-1+\mathrm{k}_{3}\left(e^{78}+79 e^{78}\right)\right)\right)^{2}}{\left(\left(\mathrm{k}_{3}\right)^{2}\left(e^{-1+e^{79}}+e^{e^{79}}\left(e^{78}+79 e^{78}\right)\right)^{2}+1\right)}\right. \\
& \left.+\frac{\left(\mathrm{k}_{3}\left(e^{-1+e^{79}}-1+\mathrm{k}_{3}\left(e^{78}+79 e^{78}\right)\right)\right) k_{3}+e}{\sqrt{\left(\left(\mathrm{k}_{3}\right)^{2}\left(e^{-1+e^{79}}+e^{e^{79}}\left(e^{78}+79 e^{78}\right)\right)^{2}+1\right)}}\right)=0
\end{aligned}
$$


The trivial solution is $k_{3}=0$ which we do not consider, because $e^{e^{e^{79}}}=k_{3}$. Further we solve the equation

$$
\begin{aligned}
& \frac{\left(\mathrm{k}_{3}\left(e^{-1+e^{79}}-1+\mathrm{k}_{3}\left(e^{78}+79 e^{78}\right)\right)\right)^{2}}{\left(\left(\mathrm{k}_{3}\right)^{2}\left(e^{-1+e^{79}}+e^{e^{79}}\left(e^{78}+79 e^{78}\right)\right)^{2}+1\right)}+ \\
& +\frac{\left(\mathrm{k}_{3}\left(e^{-1+e^{79}}-1+\mathrm{k}_{3}\left(e^{78}+79 e^{78}\right)\right) k_{3}+e\right.}{\sqrt{\left(\left(\mathrm{k}_{3}\right)^{2}\left(e^{-1+e^{79}}+e^{e^{79}}\left(e^{78}+79 e^{78}\right)\right)^{2}+1\right)}}=0
\end{aligned}
$$

By simplification we get a polynomial that has no integer or rational coefficients and therefore not even a root for $\mathrm{k}_{3}$ will not be a rational number, which means that $e^{e^{e^{79}}}$ is not a rational number, and it follows that it is not an integer.

\section{CONCLUSION}

The paper focused on the proof the Skewes' number is not an integer using lattice points and tangent line and on the problematics of prime numbers. In introduction section, we described the importance and special role of prime numbers between numbers and their importance in RSA coding. In the second section we presented algorithms Lehmann test and Rabin-Miller test which are used to determine whether a given number is a prime number and which are part of the algorithms for generating prime numbers. In this section we refer to the Prime Number Theorem, which is together with prime-counting function and logarithmic integral function described in section three. We also introduced the Skewes number in part three. The fourth section is a main contribution and showed a geometric way to prove the Skewes' number is not an integer. 


\section{REFERENCES}

[1] Garey M. R., Johnson D. S. (1990). Computers and Intractability - A Guide to the Theory of NPCompleteness. NY, USA: W. H. Freeman \& Co., ISBN 0716710455.

[2] Riesel H. (2012). Prime Numbers and Computer Methods for Factorization. Springer: New York, $2^{\text {nd }}$ ed., 482 p., ISBN 978-0-8176-8298-9, DOI 10.1007/978-0-8176-8298-9.

[3] Coutinho S. C. (1999). The Mathematics of Ciphers: Number Theory and RSA Cryptography. $1^{\text {st }}$ ed., 198 p., Brazil: A. K. Peters, ISBN: 9781568810829.

[4] Kleinjung T. et al. (2010). Factorization of a 768-Bit RSA Modulus. In: Advances in Cryptology CRYPTO 2010. Lecture Notes in Computer Science, vol 6223. Springer, Berlin, Heidelberg, DOI: 10.1007/978-3-642-14623-7_18.

[5] Sittinger, B. D. (2010). The probability that random algebraic integers are relatively r-prime. In. Journal of Number Theory, Vol. 130, No. 1, pp. 164-171., DOI 10.1016/j.jnt.2009.06.008.

[6] Duriš V. (2020). Solving Some Special Task for Arithmetic Functions and Perfect Numbers. In. 19th Conference on Applied Mathematics: proceeding, Bratislava: STU, $4^{\text {th }}-6^{\text {th }}$ of February, 2020, pp. 374 383, ISBN 978-80-227-4983-1.

[7] Lehman R. (1974). Factoring large integers. In: Mathematics of Computation, Vol. 28, No. 126, p. 637-646.

[8] Koblitz N. (1994). A Course in Number Theory and Cryptography. 2nd ed., New York: SpringerVerlag, ISBN 0387942939.

[9] Menezes J. A., Oorschot P. C., Vanstone S. A. (1997). Applied Cryptography. New York: CRC, ISBN 0-8493-8523-7.

[10] Ribenboim P. (2004). The Little Book of Big Primes. USA, NY: Springer-Verlag, 368 p., ISBN 978$0-387-21820-5$

[11] Smith D. E. (1958). History of Mathematics, Vol. I. 618p., 1st ed., US: Dover Publications.

[12] Smith D. E. (1958). History of Mathematics, Vol. II. 736p., 1st ed., US: Dover Publications.

[13] Legendre A. M. (1798). Essai sur la théorie de Nombres, 1st ed., Paris.

[14] Legendre A. M. (1808). Essai sur la théorie de Nombres, 2nd ed., Paris.

[15] Pintz J. (1980). On Legendre's prime number formula. In. The American Mathematical Monthly, Vol. 87., No. 9., pp. 733-735.

[16] Mazur B. (2016). Prime Numbers and the Riemann Hypothesis. $1^{\text {st }}$ ed., UK: Cambridge University Press, 150 p., ISBN: 978-1107499430.

[17] Hadamard, J. (1896). Sur la distribution des zéros de la fonction $\zeta(s)$ et ses conséquences arithmétiques.In. Bulletin de la Société Mathématique de France, No. 24, pp. 199-220.

[18] De la Vallée Poussin C. J. (1896). Recherches analytiques de la théorie des nombres premiers. In. Annales de la Societe Scientifique de Bruxelles No. 20, pp. 183-256.

[19] Williams H. P. (2007). Stanley Skewes and the Skewes number. In. Journal of the Royal Institution of Cornwall, ISSN 0968-5396, pp. 70-75.

[20] Abramowitz M., Stegun I. (1965). Handbook of Mathematical Functions with Formulas, Graphs, and Mathematical Tables. Revised ed., USA, Dover Publications, 1046 p., ISBN 978-0486612720.

[21] Kanwal R. P. (1996). Linear Integral Equations: Theory and technique. 2nd ed., USA Boston: Birkhäuser, 318 p., ISBN978-0817639402: .

[22] Littlewood J. E. (1914). Sur la distribution des nombres premiers. In. Comptes Rendus de l'Acad. Sci. Paris, Vol. 158, pp. 1869-1875. 
[23] Skewes S. (1933). On the difference $\pi(x)-l i(x)$. In. Journal of the London Mathematical Society, Vol. s1-8, No. 4, pp. 277-283, DOI: 10.1112/jlms/s1-8.4.277.

[24] Skewes S. (1955). On the difference $\pi(x)$ - li(x). In. Proceedings of the London Mathematical Society, Vol. s3-5, No. 1, pp. 48-70, DOI: 10.1112/plms/s3-5.1.48.

[25] Ďuriš V., Šumný T. (2019). Diophantine Geometry in Space E2 and E3. In. TEM Journal, ISSN $2217-$ 8309, Vol. 8, No. 1, pp. 78-81, DOI 10.18421/TEM81-10.

[26] Rektorys K. (1968). Overview of applied mathematics. Prague: SNTL, Language: Czech.

[27] Bronštejn I. N., Semendjaev K. A. (1961). Handbook of mathematics for engineers and for students at technical universities. Bratislava: SVTL, Language: Slovak, transl. from 8th. Rus. ed.

\author{
Viliam Ďuriš \\ Constantine The Philosopher University in Nitra \\ Tr. A. Hlinku 1, 94901 Nitra, Slovakia \\ vduris@ukf.sk \\ Timotej Šumný \\ Štefan Moyses Primary School \\ Školská 608 , Tesárske Mlyňany, Slovakia \\ sumnyt@gmail.com \\ Tomáš Lengyelfalusy \\ DTI University \\ Sládkovičova 533/20, 01841 Dubnica nad Váhom, Slovakia \\ lengyelfalusy@dti.sk
}

Received September 2021

Permission to make digital/hard copy of part of this work for personal or classroom use is granted without fee provided that the copies are not made or distributed for profit or commercial advantage, the copyright notice, the title of the publication, and its date of appear. 